\title{
Research on the circulating current suppression strategy of parallel operation of multiple cophase supply devices
}

\author{
Jing-Hua Zhou ${ }^{\dagger}$, Qiang Song, Xing -Yang Han and Yi-Kun Pan, \\ College of Electrical and Control Engineering, North China University of \\ Technology, Beijing, 100144, China; Corona, Beijing, 100000, China \\ ${ }^{\dagger}$ E-mail: zjh@ncut.edu.cn
}

\begin{abstract}
At present, the traction supply system of electrified railways has adopted phase rotation power supply technique, the power supply mode results in electrical phase-splitting of the contact line, which reduces the capacity and safety of electric locomotive. By ensuring the output voltage strict accordance of each phase power supply device, smooth cophase power supply system can be constructed, completely cancel the electric phase-splitting, but multiple cophase power supply device through the parallel power supply will bring line circulation problem. Therefore, this paper analyzes the parallel circulation problem of the electric railway in cophase power supply system, using the control theory of non reciprocal connections, a general circulation suppression strategy is proposed for the parallel operation of multiple cophae power supply devices. The simulation results show that the parallel control strategy can effectively restrain the circulating current in traction network, which can greatly improve the capacity of the line.

Keywords: Cophase Power Supply; Cascaded Multilevel; Control Strategy; Active Circulating Current Suppression.
\end{abstract}

\section{Introduction}

At present, the traction power supply system of electrified railways mainly adopts a power supply mode based on the traction transformer. Due to the use of this power supply mode, there are four major problems existing in the power supply system, including negative sequence, reactive power, harmonic wave and electric phase separation [1], which have serious impact on power quality of the electric power system and the traction power supply system, and restrict heavy-load, high-speed and economical operation of electric locomotive in the meantime [2]. However, the problems above can be effectively solved by replacing the traction transformer with the new cophase supply device in the cascaded multilevel structure. Meanwhile, through type cophase supply of the traction network can be achieved by controlling the output voltage amplitude value and phase on the cascaded output side of the new cophase supply device.

By means of the topology of cascaded energy-feedback multilevel cophase 
supply device (as shown in Figure 1), this paper puts forward a control strategy of parallel operation of multiple cophase supply devices with circulating current suppression ability. In this strategy, it is intended to adopt double-voltage closed-loop control to a single cophase supply device, and use the GPS satellite timing system to synchronize output voltage phases on the inverter side of multiple cophase supply devices. The output voltage amplitude values are uniformly given by the electric power dispatching system so that the voltage amplitude values and phases on the output side of various cophase supply devices are consistent. In addition, automatic distribution can be achieved through line impedance, so as to effectively suppress circulating current in the circuit and realize nearly power supply.

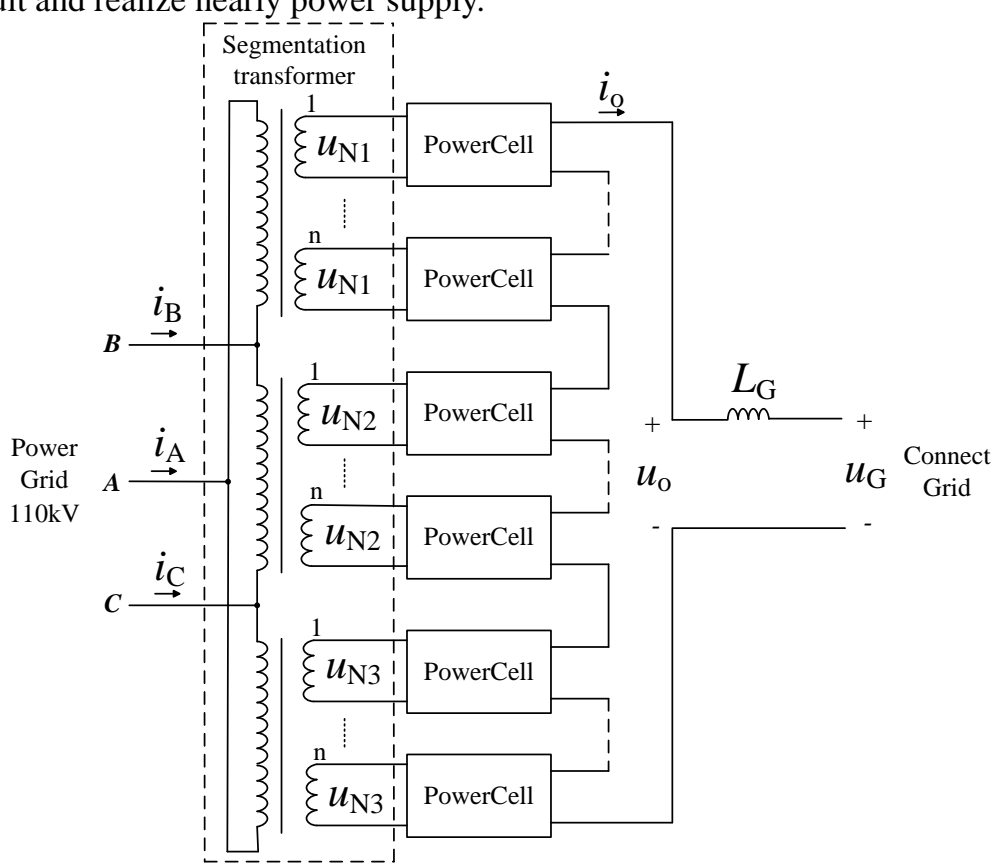

Fig.1 Topology of cascaded energy-feedback multilevel cophase supply device

\section{Generation Cause and Suppression Method of Circulating Current}

Circulating current is a key problem that must be solved for the parallel operation of multiple cophase supply devices, defined as current directly flowing from one power supply device to another without load [3]. Its existence may cause problems to the cophase supply system, such as reduction in output capacity, increase in power consumption and system oscillation. Therefore, effective measures must be taken to suppress circulating current.

A parallel simplified circuit of two cophase supply devices is analyzed below: 


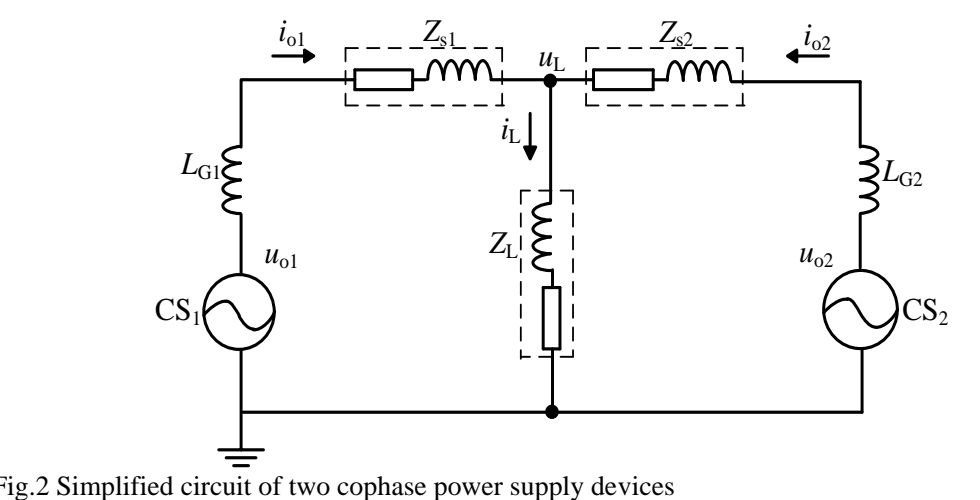

Fig.2 Simplified circuit of two cophase power supply devices

In a cophase supply system without a traction transformer, the existence of line impedance leads to both series load and parallel load in output load. $\boldsymbol{I}_{\mathbf{0 1}}-\boldsymbol{I}_{\mathbf{0} 2}$ cannot accurately represent "current flowing from one inverter to another without load".

Thus, in a remote distributed parallel power supply system, there are two methods for current suppression in the condition of constant impedance in the whole line [4].

(1)Increase current-limiting inductance $L_{G}$;

(2)Decrease output voltage difference among various cophase supply devices.

\section{Active Circulating Current Suppression Strategy of Parallel Operation of Multiple Cophase Supply Devices}

\subsection{Theoretical analysis}

Considering the difference in physical parameters of feedback channels of various cophase supply devices without the traction transformer, there are error coefficients $k_{\mathrm{s} 1}$ and $k_{\mathrm{s} 2}$ in feedback channels $u_{\mathrm{o} 1}$ and $u_{\mathrm{o} 2}$, as shown in Figure 3. Inconsistent feedback error coefficients of various cophase supply devices may directly cause errors of $u_{\mathrm{o} 1}$ and $u_{\mathrm{o} 2}$, and further lead circulating current to the parallel power supply system. Thus, circulating current must be suppressed.

When the system is loaded, $i_{\mathrm{H}}$ cannot be extracted in the way of $i_{\mathrm{o} 1}-i_{\mathrm{o} 2}$, and it is impractical to acquire locomotive position and instantaneous input current in real time in an actual traction power supply system. However, circulating current does not vary with load conditions for remote distributed voltage-source parallel systems, in which line impedance cannot be ignored, such as cophase supply devices. Therefore, it can be ensured that there is no circulating current generated in a loaded parallel system by locking the constant output voltage 
amplitude value and phase without circulating current in the no-load condition.

When the parallel system is non-loaded, its parallel model can be simplified to a model shown in Figure 3.

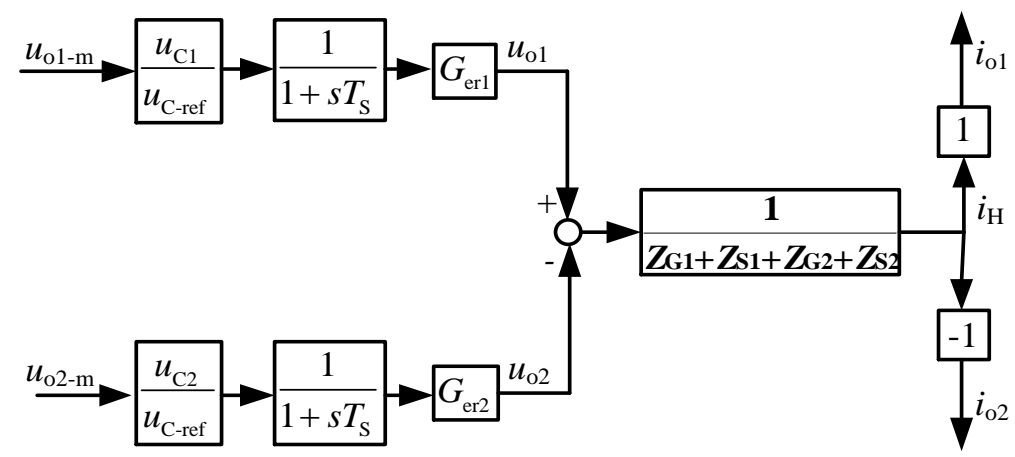

Fig.3 No-load physical model diagram of parallel operation of cophase power supply systems without traction transformer

In Figure 3, $u_{\mathrm{o} 1-\mathrm{m}}$ and $u_{\mathrm{o} 2-\mathrm{m}}$ are reference modulating voltage values of $\mathrm{CS}_{1}$ and $\mathrm{CS}_{2} ; u_{\mathrm{C} \text {-ref }}$ is the sum of set voltage values of the unit DC bus, as well as the amplitude value of carrier wave on the inverter side. $u_{\mathrm{C} 1}$ and $u_{\mathrm{C} 2}$ are respectively the sums of actual voltage values of the unit DC bus of $\mathrm{CS}_{1}$ and $\mathrm{CS}_{2}$. $G_{\text {er1 }}$ and $G_{\text {er2 }}$ are error transfer functions added in order to represent the actual physical difference between two cascaded inverters. $Z_{\mathrm{s} 1}$ and $Z_{\mathrm{s} 2}$ are line impedance of two parts. $Z_{G 1}$ and $Z_{G 2}$ are output inductances of inverters.

When the system is non-loaded, $Z_{\mathrm{L}}$ is infinite, and the reference direction of circulating current is selected as $i_{\mathrm{o} 1}=i_{\mathrm{H}}$ and $i_{\mathrm{o} 2}=-i_{\mathrm{H}}$. In this case, a single new cophase supply device can effectively extract circulating current and lead it to the control system, and enable $i_{\mathrm{H}}=0$ by adjusting the output voltage amplitude value and phase of the device, so as to achieve active circulating current suppression for the system.

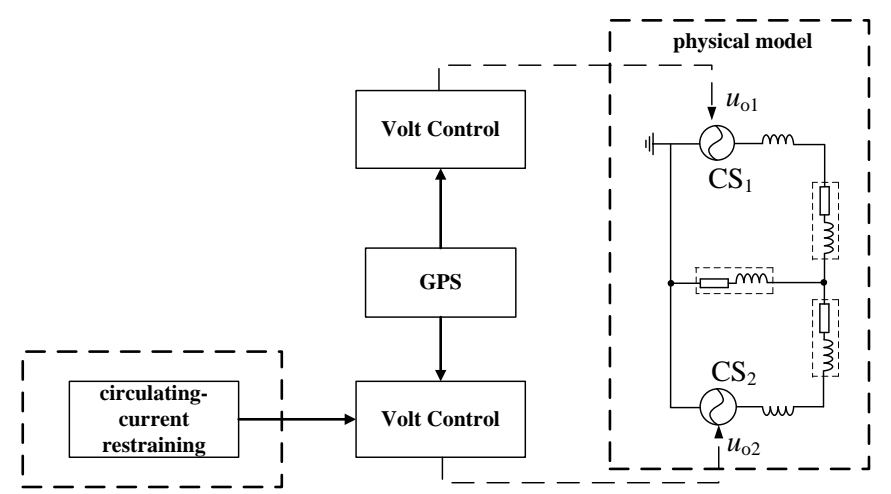

Fig.4 Control block diagram of parallel power supply system with circulating current suppression 
Providing $\mathrm{CS}_{1}$ is firstly put into the traction network and used for supplying power to the traction network independently, and the this section of traction network is non-loaded, the control strategy of active circulating current suppression shall be implemented as soon as $\mathrm{CS}_{2}$ is also put into the traction network so that output current of $\mathrm{CS}_{2}$ can be 0 . Through output current calibration of the latter device put into the network, this strategy completely equalizes output voltages of $\mathrm{CS}_{2}$ and $\mathrm{CS}_{1}$, and locks the output voltage amplitude value and phase in the meantime, so as to realize the ultimate objective of thorough circulating current suppression for the system.

In the no-load condition of traction power supply system, the control strategy of active circulating current suppression shall be implemented in order for other power supply devices by taking a certain new cophase supply device as a reference of the system. Corresponding to Figure 2, when $\mathrm{CS}_{1}$ works normally, $\mathrm{CS}_{2}$ implements the control strategy of active circulating current suppression, accurately calibrate output currents, and ultimately make output voltages of $\mathrm{CS}_{2}$ and $\mathrm{CS}_{1}$ completely equal to those of $u_{02}$ and $u_{\mathrm{o} 1}$, so as to achieve active circulating current suppression. At the moment, out currents of the two power supply devices are all 0 . After judging $i_{\mathrm{o} 2}$ to 0 through the control system, $\mathrm{CS}_{2}$ immediately locks the current phase compensation $\varphi_{\text {off }}$ and amplitude value

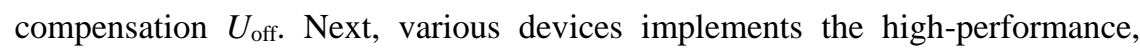
multi-loop and composite control \& dispatching strategy on the inverter side without current loop. After this section of contact network, the loaded system can operate normally without circulating current, and so forth. In addition, when a certain section of contact network is in a no-load condition during operation of system, the control strategy of active circulating current suppression can be restarted to recalibrate circulating current in this section of contact network, which has no impact on normal power supply in the contact network owing to the very short implementation time of this control strategy.

\section{Simulation Result and Analysis}

During the simulation of parallel operation of two devices, set the distance between the two cophase supply devices to $30 \mathrm{~km}$, and place the locomotive in the $1 / 4$ position where it is $7.5 \mathrm{~km}$ away from the first device and $22.5 \mathrm{~km}$ away from the second device. Upon the completion of simulation, the result is shown in Figure 5. 


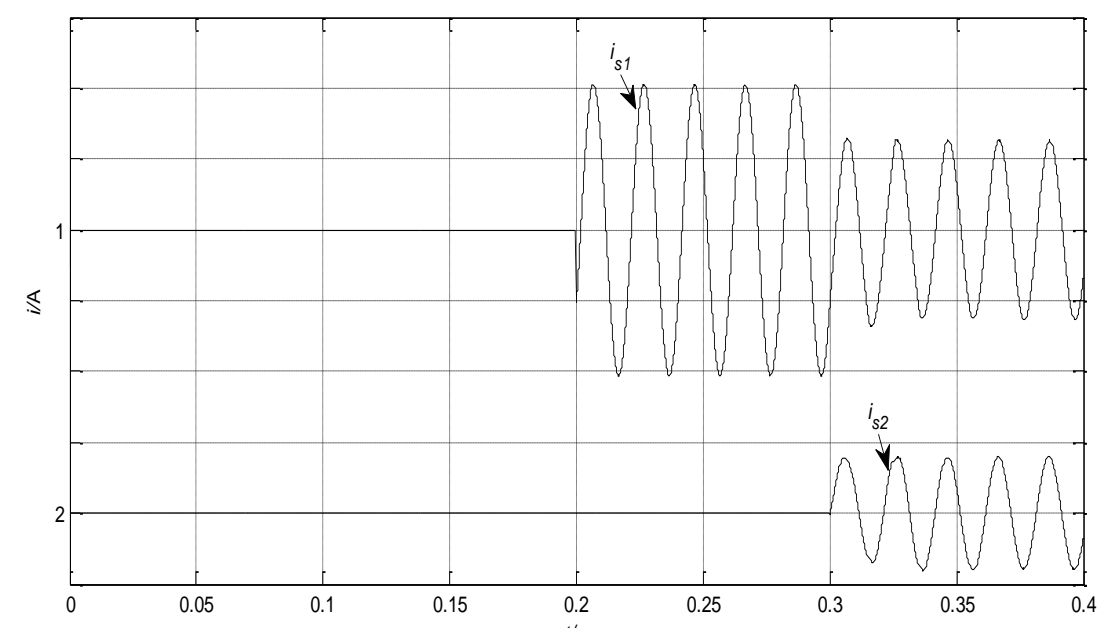

Fig.5 Output current waveforms when load is located in the $1 / 4$ position of two devices

As shown in Figure 5, the y-coordinate is set at an interval of 500A, and the $\mathrm{x}$-coordinate is set at an interval of $0.05 \mathrm{~s}$. At $0.2 \mathrm{~s}$, only the first device is put into operation in the contact network with output current of $1000 \mathrm{~A}$. At $0.3 \mathrm{~s}$, the second device is put into operation in the contact network. This moment, output current of this first device is 600A, and output current of the second device is 400A. The sum of output currents is 1000A. There is slight phase difference between output currents of the two devices, but no circulating current between the two devices.

During the simulation of parallel operation added with the third device, load is located in the middle of the first and second devices. Set the distance between each two devices to $30 \mathrm{~km}$, and place the locomotive in the middle of the first and second devices where it is $15 \mathrm{~km}$ away from both the first and second devices and $45 \mathrm{~km}$ away from the third device. Put the first device into operation at $0.2 \mathrm{~s}$, put the second device into operation at $0.3 \mathrm{~s}$, and put the second device into operation at $0.4 \mathrm{~s}$.

Figure 6 shows the output current waveforms in parallel operation of three devices when load is located in the middle of the first and second devices. 


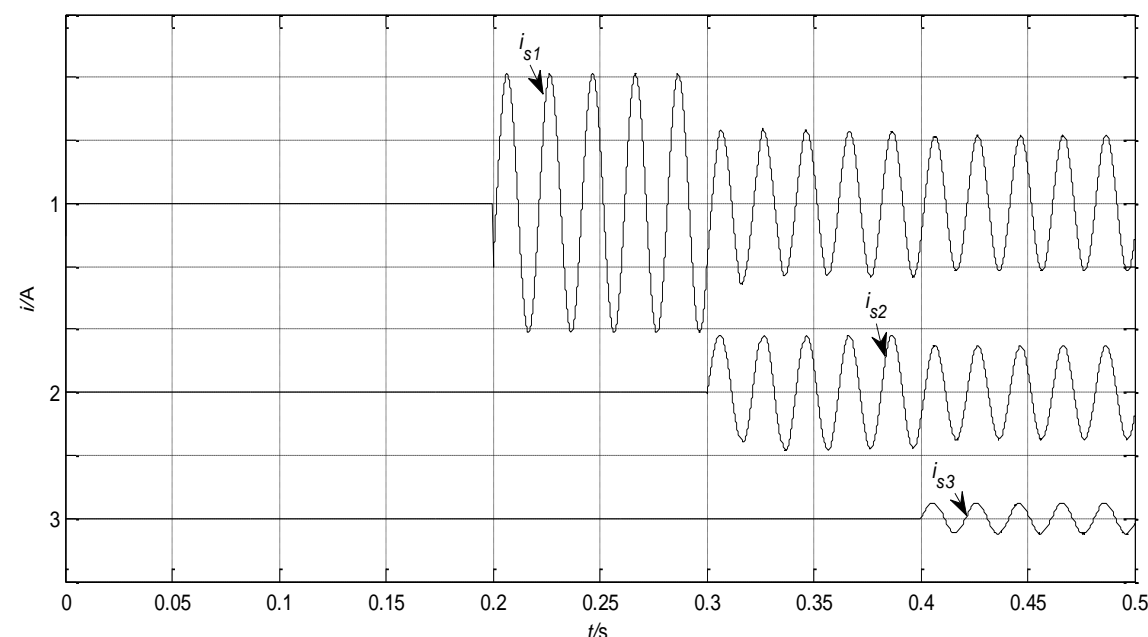

Fig.6 Output current waveforms in parallel operation of three devices when load is located in the middle of the first and second devices

In figure 6 , the $\mathrm{y}$-coordinate is set at an interval of $500 \mathrm{~A}$, and the $\mathrm{x}$-coordinate is set at an interval of $0.05 \mathrm{~s}$. It can be learned from the figure that when all the 3 devices are put into operation in the contact network, output current of this first device is $500 \mathrm{~A}$, output current of the second device is $375 \mathrm{~A}$, and output current of the third device is 125A. Output currents of the 3 devices are distributed in proportion according to the total output current. There is no circulating current among the power supply devices. In addition, a device in a relatively short distance from load is distributed with large current, while a device in a relatively long distance from load is distributed with small current. Thus, the system follows the principle of nearby power supply.

\section{Conclusion}

Based on the topological structure of energy-feedback cascaded multilevel cophase supply device, this paper puts forward a control strategy of parallel operation of multiple cophase supply devices with active circulating current suppression ability. As verified through simulation, this control strategy of parallel operation can achieve strictly consistent output voltage amplitude values and phases on the inverter side of multiple cophase supply devices, so as to effectively suppress circulating current in the system and make through-type power supply possible, which can significantly improve operating speed and heavy-load ability of electric locomotives, reduce the overall operating cost, and provide some theoretical bases for further construction and development of the traction power supply system of electrified railways in the future. 


\section{Acknowledgement}

This work is supported by the Beijing Natural Science Foundation of China (3142008).

\section{References}

1 Shu ze-liang, Xie shao-feng, Li Qun-zhan. Single-phase back-to-back converter for Active Power Balancing. Reactive Power Compensation and Harmonic Filtering in Traction Power System [J]. IEEE Transactions on Power Electronics, 2011, 26(2):334-343.

2 Xiaoqiong He, Zeliang Shu, Xu Peng, et al. Advanced Cophase Traction Power Supply System Based on Three-Phase to Single-Phase Converter [J]. IEEE Transactions on Power Electronics, 2014, 29(10):5323-5332.

3 Liu Y, Ge B, Abu-Rub H, et al. An Effective Control Method for Quasi-Z-Source Cascade Multilevel Inverter-Based Grid-Tie Single-Phase Photovoltaic Power System [J]. IEEE Transactions on IndustrialInformatics, 2014, 10 (1):399-407.

4 Shu Z L, Xie S F, Lu K, et al. Digital Detection, Control, and Distribution System for Co-phase Traction Power Supply Application [J]. IEEE Trans on Industrial Electronics, 2013, 60(5):1831-1839.

5 Zhang X, Spencer J W, Guerrero J M. Small-Signal Modeling of Digitally Controlled Grid-Connected Inverters With LCL Filters [J]. IEEE Transactions on Industrial Electronics, 2013, 60(9):3752-3765. 